\title{
Similarities between hurricanes and galaxies: A short review
}

\author{
Jim Henry ${ }^{1}$, Mesut Yurukcu ${ }^{2}$, and George Nnanna ${ }^{2}$ \\ ${ }^{1}$ Henry Resources, Midland, TX, 79703 \\ ${ }^{2}$ University of Texas Permian Basin, The College of Engineering, Midland, TX, 79705 \\ *E-mail of the corresponding author: yurukcu_m@utpb.edu
}

\section{Highlights}

- The comparison between hurricanes and galaxies.

- Review article about similarities between hurricanes and spiral galaxies.

\begin{abstract}
Universe created with the fundamental laws of science. Nature is lazy and needs to form with the least possible to be perfect. A natural pattern, such as pinecones, sunflowers, pineapples, and cacti, has a double spiral structure. Once we look at these plants' centers, we will see the seeds line up in spirals shape. The number of spirals whirling in each direction will give us the Fibonacci numbers. We can give more examples representing these natural patterns; however, one example is unique and remarkable. The similarities between spiral galaxies- Milky Way and hurricanes. Are they similar in every property or just in shape and rotational movements? What are the similarities between them?
\end{abstract}


This short review article will try to find these questions' answers by reviewing some literature articles. The first part of this article gave some information about hurricanes and galaxies. The second of this article focused on the comparison between hurricanes and galaxies. Finally, we will conclude the article with our remarks.

Keywords: Universe, galaxies, Milky Way Galaxy, spiral galaxies, hurricanes, pattern.

\section{Introduction}

Human beings have been looking for answers about the universe for centuries. In every century, physicists and cosmologists were asking many questions and trying to find their answers. Our planet, Earth, is one of the planets inside Solar Systems in our galaxy. Once we launched the telescopes to space, we collected more real images and data related to space (de Miguel et al., 2021). One of these data belongs to the galaxies (Morishita 2021). What are their shapes? How did they form? On the other side, with the new technology, we can collect Earth's photos and data. Significantly, hurricanes can be seen from space how they form and move. We can develop one common question when putting the galaxies' and hurricanes' images side by side. Are galaxies and hurricanes the same? Galaxies and hurricanes have some similarities, such as shape and movement; however, the formation and properties are entirely different. Galaxies have different types for their shape (Cao et al., 2018). Only spiral galaxies have similarities with hurricanes. Most galaxies are spinning around a central point, which can be their supermassive black hole (Li, E. 2016). The spiral arms of the galaxies are becoming from concrete structures like stars. While the galaxies' arms, which stars rotate, the arms would be stretched out as they were in a giant cosmic taffy machine (Hofmeister et al., 2017b). 
Hurricanes are very similar to the galaxy in shape and movement (Cao et al., 2018). Many scientists want to learn from these similarities to get more information about galaxies by using the hurricane. To the best of our knowledge, galaxies and hurricanes have similarities only in shape and movement. Hurricane is a disturbance in a uniform medium. In contrast, a galaxy is a collection of matter in a vacuum with a concentrated mass in its center (Sánchez 2020), and a hurricane has low pressure in its center. We compared the similarities and contrasts between hurricanes and galaxies, followed by brief details about galaxies and hurricanes in this article.

\section{Galaxies}

A galaxy in which gravity holds the galaxies together consists of dust, gas, billions of stars. The middle of the galaxies has a supermassive black hole (Feng 2020). Astronomers classified the Galaxies for their shapes, such as spiral, elliptical, lenticular, or irregular (Figure 1) (NASA 2009).

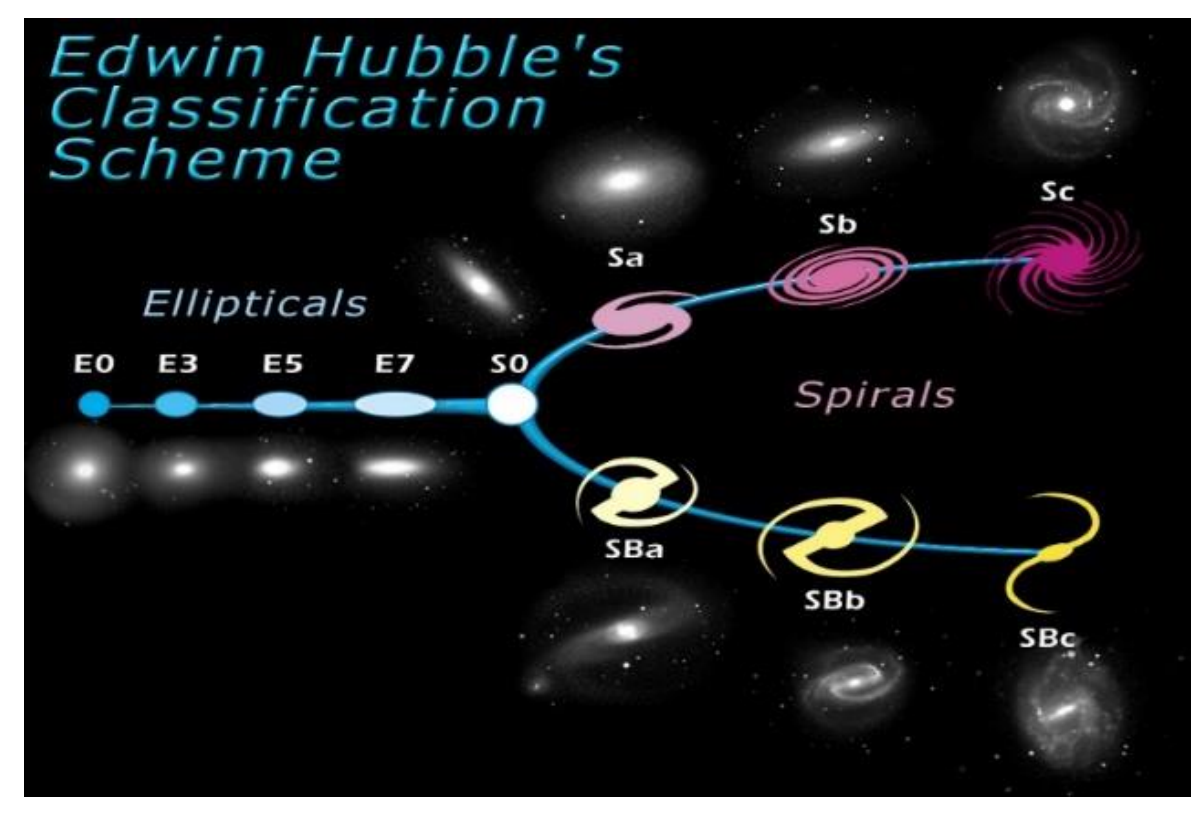

Figure 1: Edwin Hubble's Classification Scheme (Image Credit to NASA 2009) 
In spiral galaxies, stars, gas, and dust clouds are organized in a bulge, a halo, and a disk (Figure 2). Cao et al. showed that quasi-steady solutions could be obtained from the gravitational field equations and dynamical equation of galaxies to explain the galaxies' spiral structure (Cao et al., 2018). They proposed that the spiral galaxies are not isolated systems, but the background's motion drives their evolution. Thus, the spiral structure is a non-inertial force evoked from the participants' universal background during the galaxies' creation (Cao et al., 2018).

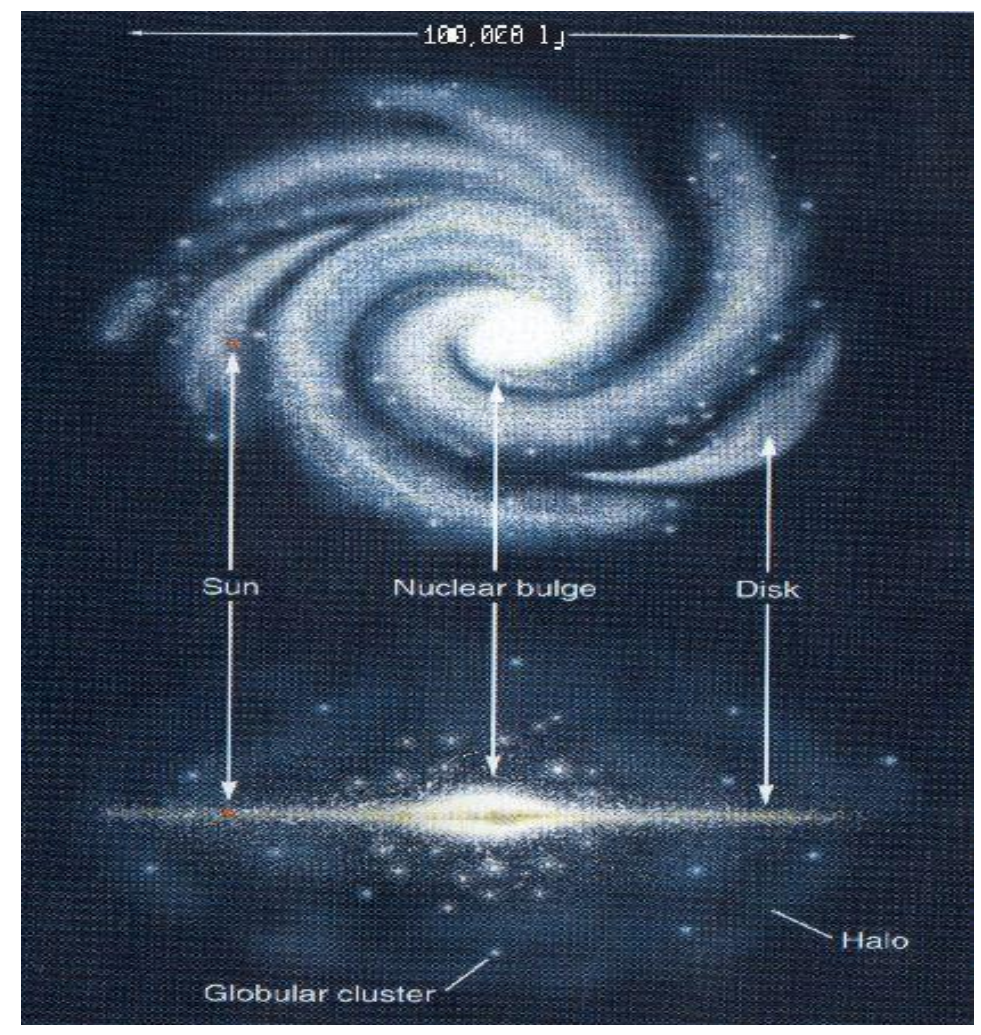

Figure 2: A Milky Way Galaxy consisting of the parts (Image Credit to Smith 1999).

Elliptical galaxies have a halo and bulge (a round structure of old stars, gas, and dust) rather than a disk. Milky Way's bulge size is around 10,000 light-years across (English 2014). The disk of galaxies is flattened and has a rotating part that includes Sun and other young stars. Disks have atomic and molecular gas and dust. Spiral arms can be found only in spiral galaxies (Figure 3). 
The arms curve outward, which shows the galaxy as a pinwheel. Another part of the galaxies in the halo. Halo contains dark matter, the oldest stars with a little bit of hot, highly ionized gas and dust(Criss et al., 2018), which size larger than 130,000 light-years across (Dorminey 2002).

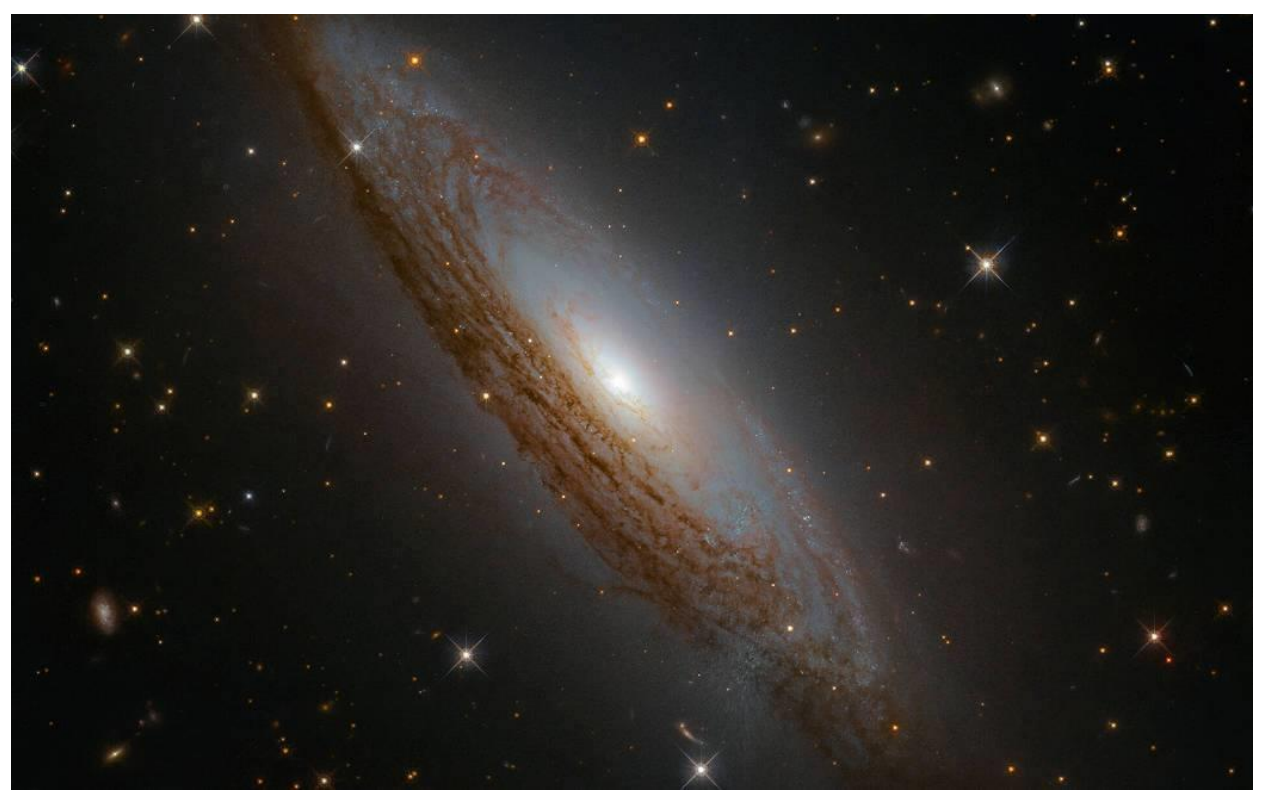

Figure 3: This swirling mass of celestial gas, dust, and stars is a moderately luminous spiral galaxy named ESO 021-G004, located just under 130 million light-years away (Image Credit to NASA/Hubble 2019).

Stars in galaxies have a variety of types, such as blue and red stars (Davidge 2021). Blue stars are scorching and have shorter lifetimes than red stars. Spiral galaxies have more gas and dust compared to Elliptical galaxies. For example, our galaxy, Milky Way Galaxy, has a spiral shape galaxy. When people think about the galaxies, the spiral-type galaxies come to mind due to the Milky Way Galaxy type (Criss et al., 2020). Spiral galaxies rotate like a hurricane (Figure 4). 


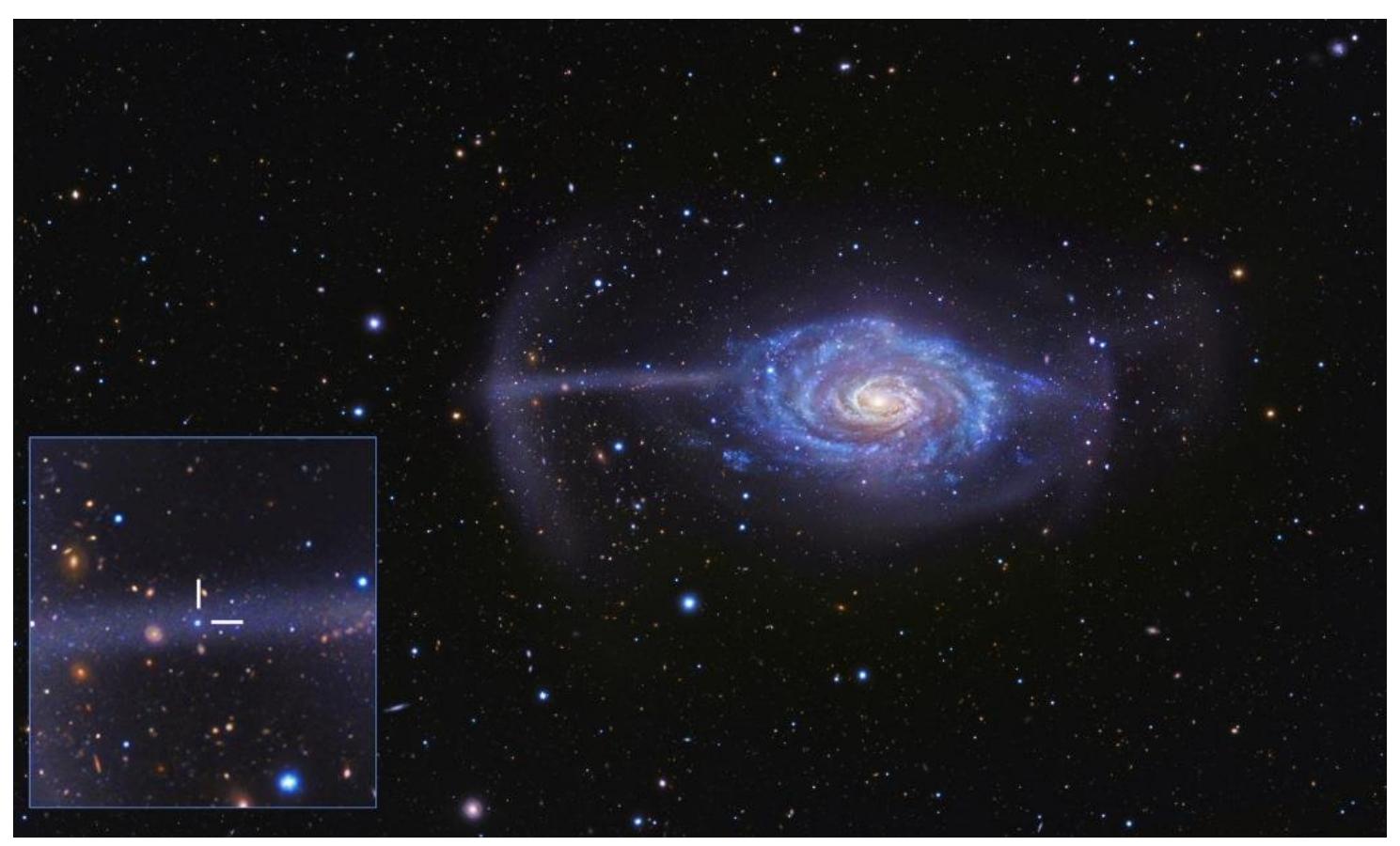

Figure 4: A spiral galaxy, known as NGC 4651, swirls in the vast, silent emptiness of space (Image Credit to NASA \& R. Jay Gabany 2014).

There are several explanations for the rotation of galaxies. One of them is spiral arms driven by the large bar shape at the center of the spiral galaxies (Figure 5). Another explanation is stochastic self-propagating star formation. In this explanation, star formations can cause a shock wave; stretch the new stars in spiral arms, and burn out the old stars to make a reaction, so the arms move on (Suntola 2013). 

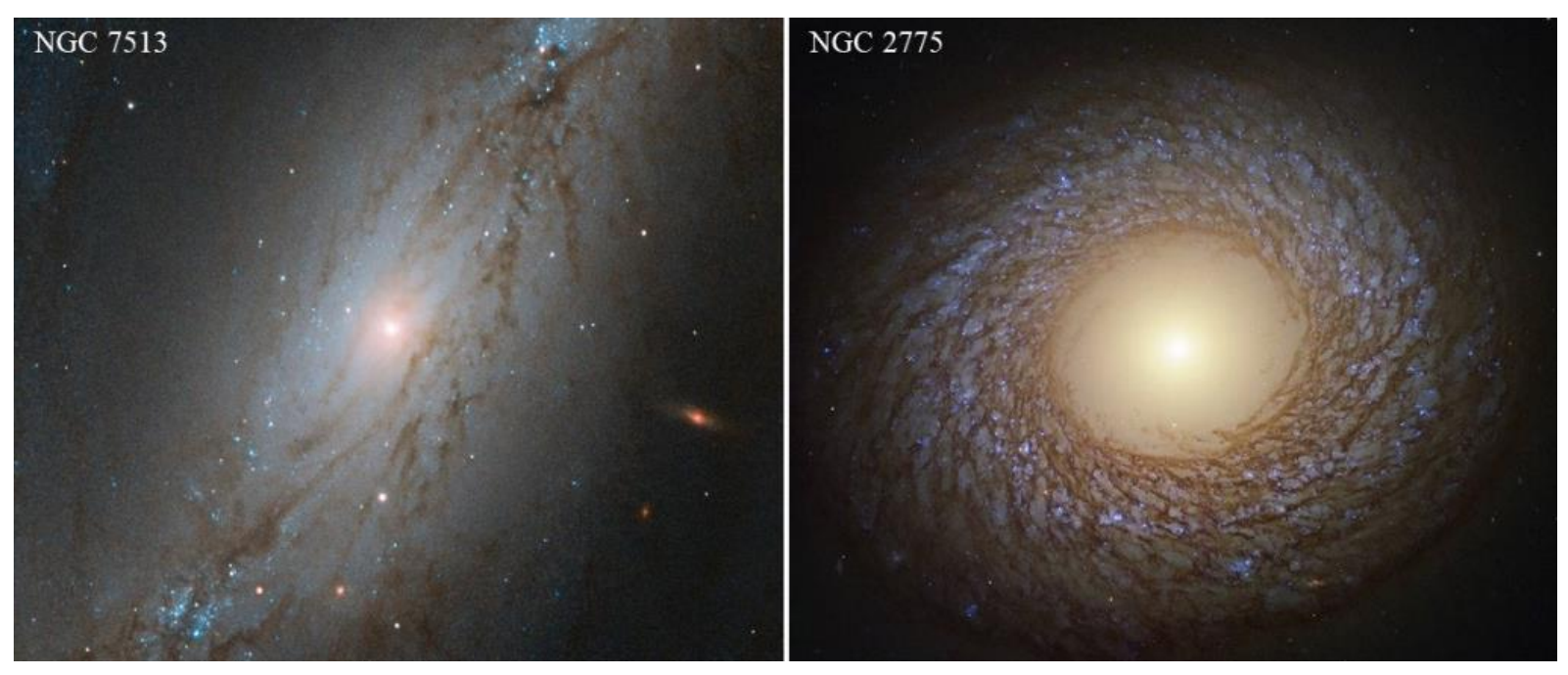

Figure 5: Hubble Space Telescope captured two spiral shape galaxies; NGC 7513 (left image), a barred type of spiral galaxy, and NGC 2775 (right image) (Image Credit to NASA \& ESA/Hubble 2020).

Another galaxy type is an elliptical shape galaxy (Figure 6). Elliptical galaxies have a ballshaped and smooth appearance instead of having a disk or spiral arms, which contain old stars and gas (McDaniel et al., 2021). To classify them, their shape is a ball, like baseball shaped. The most common type of galaxies is the smallest elliptical galaxies around our galaxy. In contrast to spirals, the stars move in randomly oriented orbits within the galaxy (Criss, et al., 2018).

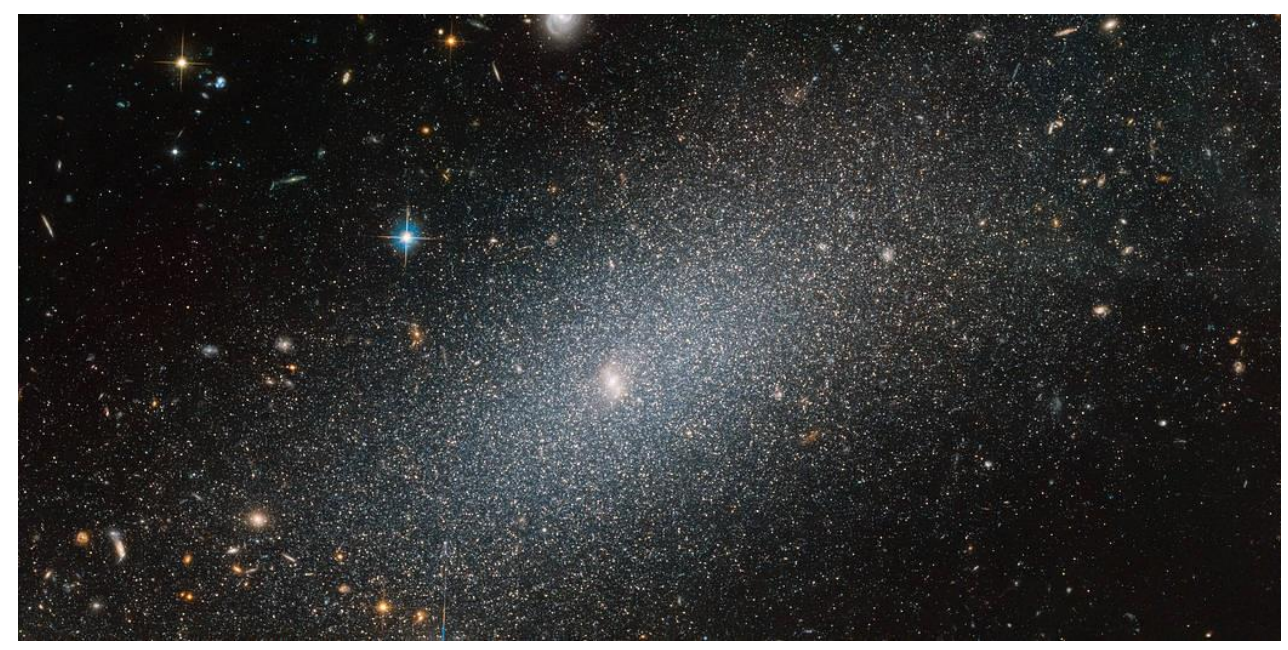


Figure 6: A spotlight-hogging Galaxy, PGC 29388, is a dwarf elliptical galaxy (Image Credit to NASA\& ESA/Hubble \&T. Armandroff, 2020).

Irregular galaxies are shaped by the result of interactions with other galaxies. They have mixed dust, newborn stars, and gas to form active regions of the star. Hubble Deep Fields' observations show that irregular galaxies were more common in the distant universe(Williams 1996). The comparison of these three types of galaxies can be found in Table 1.

Table 1: Comparison of the Galaxies

\begin{tabular}{|c|c|c|}
\hline \multicolumn{3}{|c|}{ Main Classes Galaxies } \\
\hline $\begin{array}{c}\text { Spiral Galaxies } \\
\text { (Fernandez. Et. Al., 2021) }\end{array}$ & $\begin{array}{l}\text { Elliptical Galaxies } \\
\text { (Shah et al., 2021) }\end{array}$ & $\begin{array}{c}\text { Irregular Galaxies } \\
\text { (Abdallah, et al., 2021) }\end{array}$ \\
\hline $\begin{array}{l}\text { Contain stars, gas, and dust. } \\
\text { They are huge. }\end{array}$ & $\begin{array}{l}\text { Contain stars, gas, and dust. } \\
\text { They are huge. }\end{array}$ & $\begin{array}{l}\text { Contain stars, gas, and dust. } \\
\text { They are huge. }\end{array}$ \\
\hline Held together by gravity & Held together by gravity & Held together by gravity \\
\hline Pinwheel shape & Round to oval shape & No regular shape \\
\hline $\begin{array}{l}\text { Bulge and think disk; halo is } \\
\text { present }\end{array}$ & $\begin{array}{l}\text { Bulge but no disk. A halo is } \\
\text { present }\end{array}$ & $\begin{array}{l}\text { May show signs of a disk } \\
\text { and/or a bulge; halo is present }\end{array}$ \\
\hline Rich in gas and dust & $\begin{array}{l}\text { A small amount of cool gas and } \\
\text { dust }\end{array}$ & Usually rich in gas and dust \\
\hline $\begin{array}{l}\text { Young and old stars are } \\
\text { present }\end{array}$ & Mainly old stars are presents & $\begin{array}{l}\text { Young and old stars are } \\
\text { present }\end{array}$ \\
\hline
\end{tabular}




\section{Hurricanes}

A big hurricane is a rounded shape. The winds of a hurricane increase precipitously to the highest from the center (Galindo et al., 2021). Then winds slower toward the storm's outer extent. The size of a hurricane's wind could be around a hundred miles across (Chang et al., 2021). On the other side, a tropical storm wind size maybe around 300 miles greater than the eye of a large hurricane. On October 12, 1979, near the Northwest Pacific Ocean, one of the largest tropical storms occurred and was recorded at a diameter of about 1350 miles. Cyclone Tracy (Darwin, Australia, December 24, 1974) was one of the smallest tropical cyclones was measured about 60 miles across (West 2000).

The eyewall, outer region, and eye are the three main parts of a big hurricane. Strong surface winds move inside the storm's center and make a piece of serene air for the huge hurricanes (Devaraj et al., 2021). The center of the hurricane is so clear that you can see the Sun or stars very neat. On the other side, the outside of the center of the hurricane is surrounded by a ferocious, stormy eyewall, which formed the strongest winds and heaviest precipitation (Ramage 1959).

The rainbands produce heavy rain and wind, which spiral inwardly in the direction of the eyeball. Occasionally, gaps can occur between the bands and end up without rain (Stow et al., 2020). However, if one of the bands moves from the outer to the inner edge, the light rain can be turned into heavy rain many times. This rainfall could be more powerful and last longer until arriving at the eye of the hurricane. If the eyewall occurs just before the landfall, alternate cycles can have severe effects (Wu et al., 2003). In 1992, hurricane Andrew near Florida increased power to category five and destroyed properties and life. Its eyewall is replaced with a cycle, increasing its huge and speedy intensity swings (Willoughby et al., 1996). 


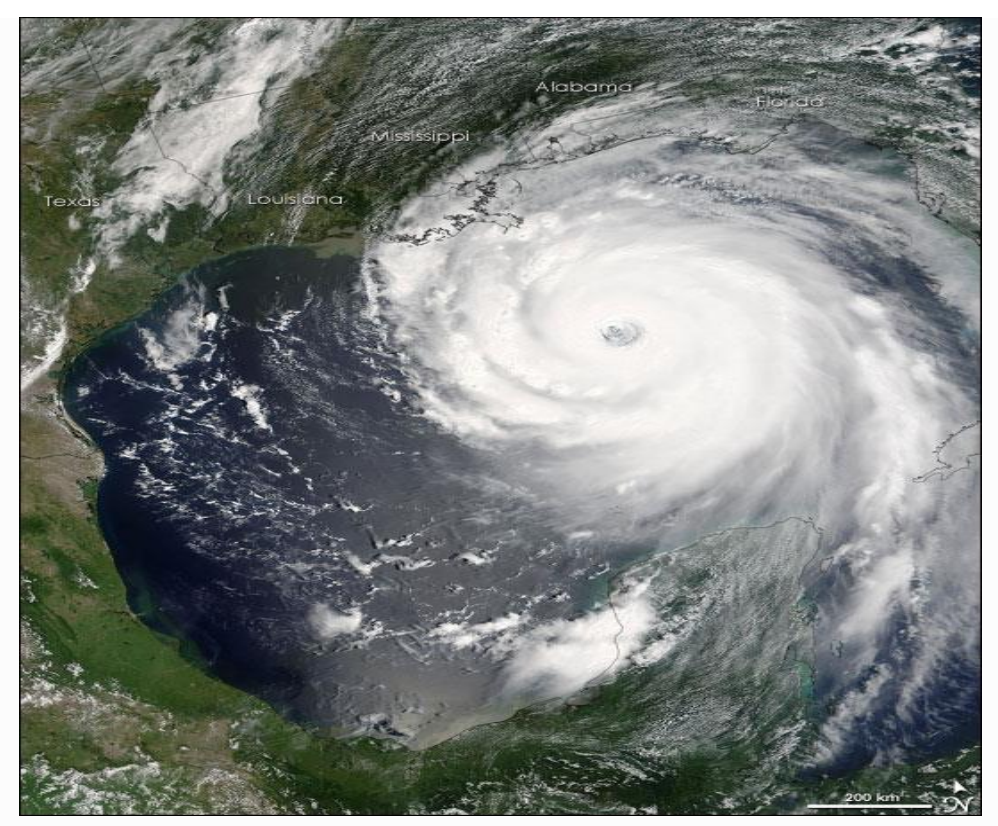

Figure 7: Hurricane Katrina (NASA, 2005)

For example, Hurricane Katrina (Figure 7) threatened New Orleans with a larger and dangerous storm when it moved through the Gulf of Mexico. When the hurricanes become bigger and stronger, their wind damage is more followed by huge storm surge and wave heights because wind fetches increased. The hurricane can grow larger every time several eyewalls are replaced with cycles (Brunkard et al., 2008). A good example of this replacement in a hurricane is Hurricane Igor (2010), one of the larger Atlantic hurricanes. It caused huge waves and ripped currents along the U.S. east coast (Han et al., 2012).

Furthermore, hurricane eyes are could also be oblong shapes (Johnson et al., 2019). However, elliptical eyes are noted mostly in slower hurricanes (Zhang et al., 2016). The shape of the eye could be square, pentagon, or triangle if the hurricane has polygonal eyewall associated with smaller-scale atmospheric swirls (Houze et al., 2007). One of the still-hot topics about hurricanes is how galaxies form. Hurricanes need a combination of atmospheric processes that low 
pressure inside the eye causes contraction or attraction. They have a lifetime, same as stars, such as grow, mature, and vanish. The rotation of the Earth and the inertia of the air masses cause the expansion of the hurricanes (Latif et al., 2007).

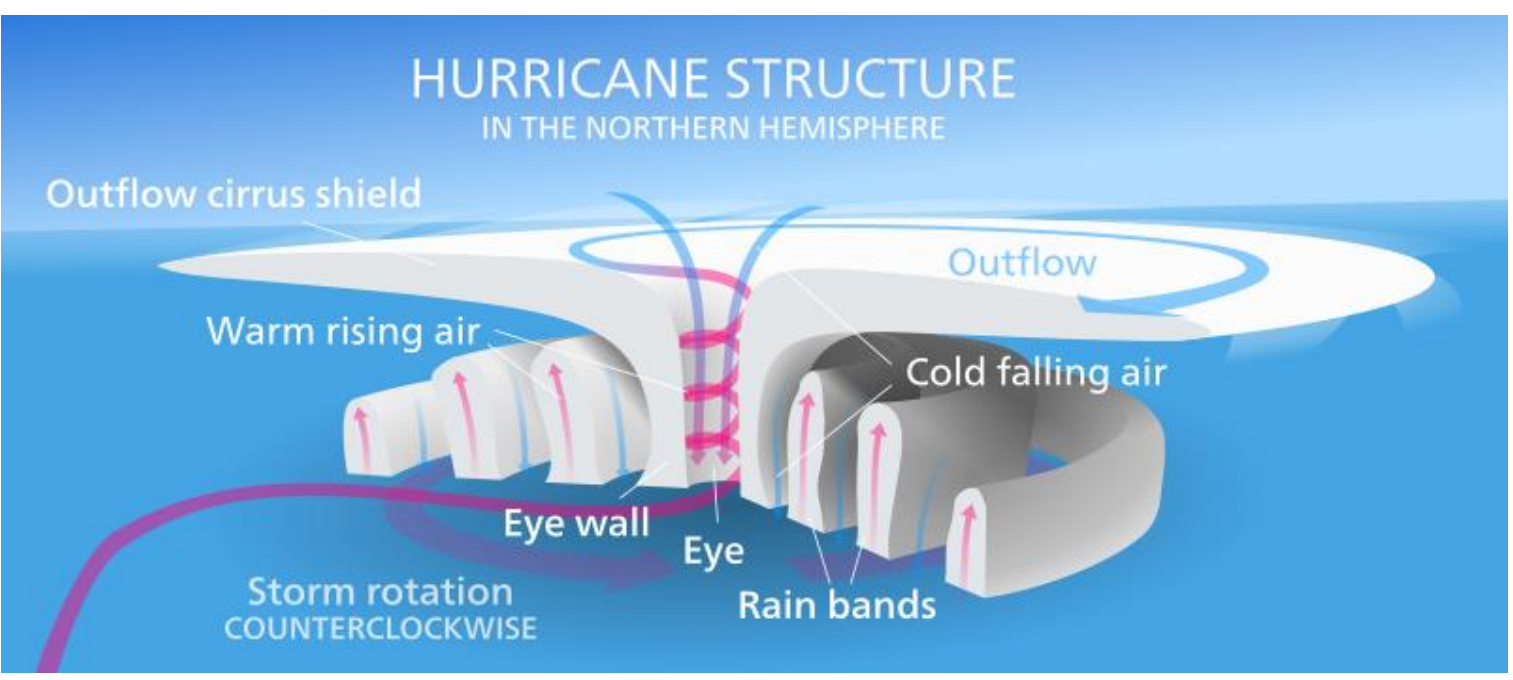

Figure 8: An illustration of a hurricane (Image Credit to Kelvinsong 2012).

Heavy spiral winds with inward-cold air move from above sinks into the calm cloud-free eye. A hot moist air, including rain bands from the surface, causes condensation into drops at the bottom of the hurricane. The warm air spirals upwards to the eyeball with the reason of condensation that releases additional energy and more heat. The air pressure at the surface drops when the warm air rises. The large difference in pressure between the center and the surrounding field causes strong winds and sucks more air into the core (Collins 2006). At the top, the air spreads out over the storm (Figure 8) (Kelvinsong 2012). As a result, hurricanes and galaxies form an unorganized cluster of thunderstorms, and stars, respectively. Hurricanes form over tropical oceanic regions when sea surface temperature reaches $26 \mathrm{C}^{\circ}$ with Earth's spinning circulation, low pressure, moist air, and tropical wind patterns (Kossin 2017). 


\section{Comparison for hurricanes and spiral galaxies (Milky Way Galaxy)}

Both hurricanes and spiral galaxies rotate, and one ring forms around the central disc with each completed rotation (Figure 9). The center of hurricanes is a calm area with fewer clouds than the edges, while the center of galaxies has more stars than the edges (Lehnert 1996). The only differences between their rings are made of them. The hurricane ring made from water gas and galaxies is made of stars and planets with solid and frozen matter (Kaiser et al., 1992). Because of frame dragging and conversation of energy, galaxies have a flat spiral shape. Hurricanes have a spiral shape because of temperature differences and the Coriolis force effects(Cao et al., 2018). The wind begins to flow toward its center and near the surface when an area of low atmospheric pressure forms (Emmanuel 1987).

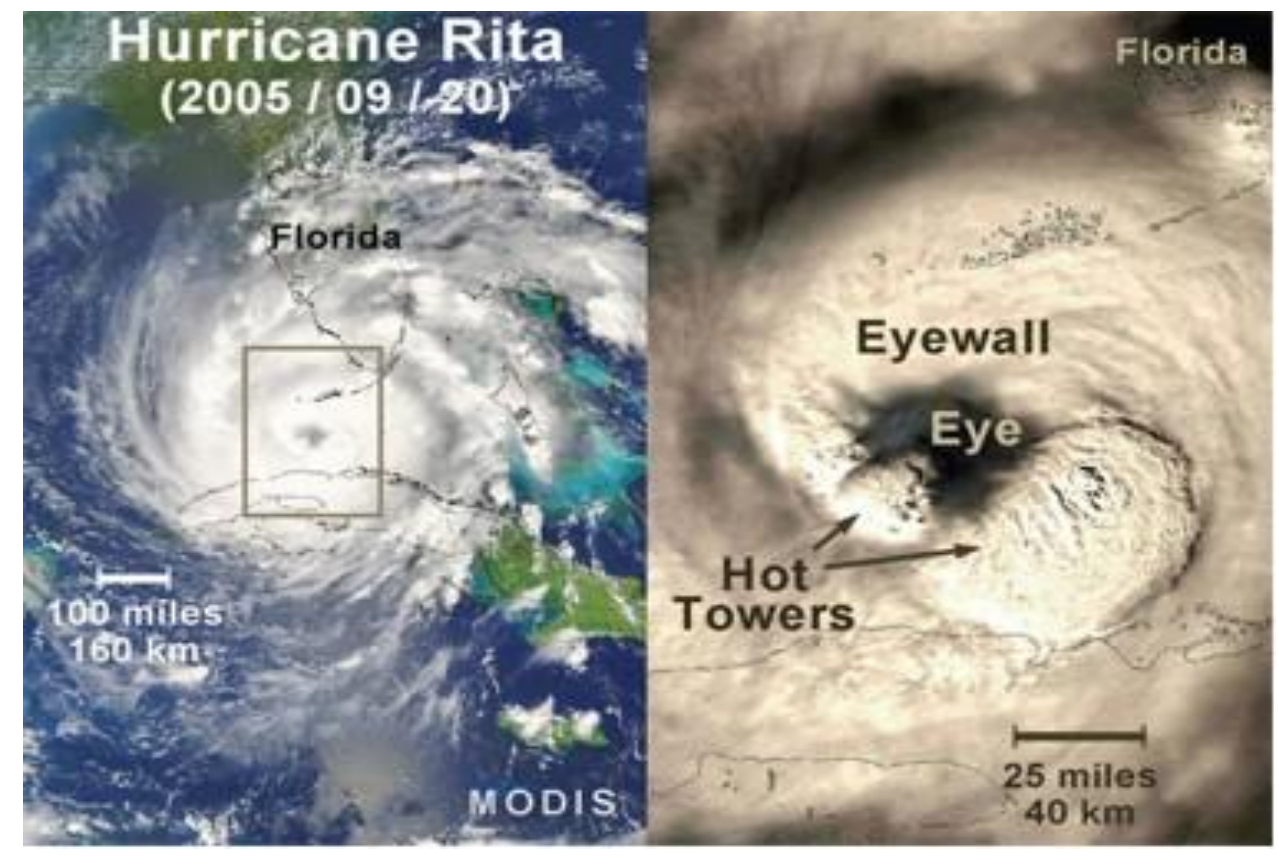

Figure 9: Satellite view (MODIS) and detailed imagery of Hurricane Rita as she intensified on September 20,2005. The area contained in the square on the left is depicted to the right. The cloudfree eye and surrounding eyewall are visible (Image credit to Gutro \&NASA 2006). 
Storms south of the equator rotate clockwise (Gibbons 2020). The galaxies' curvature caused by gravity motivates matter, whereas a hurricane is more affected by different atmospheric temperatures (Lineweaver et al., 2008). Galaxies with bulging centers are flat spiral arms due to rotation and density waves (Feng 2020). Hurricanes are Earth's gravitational field structures, while galaxies are self-gravitating objects in space (Criss et al., 2018) with galaxy density. Satellites of the galaxies move indefinitely with galaxy's density waves (Hofmeister et al., 2017b). Astronaut Ed Lu took this impressive photo (Figure 10) below from Hurricane Isabel's eye from the International Space Station in September 2003.

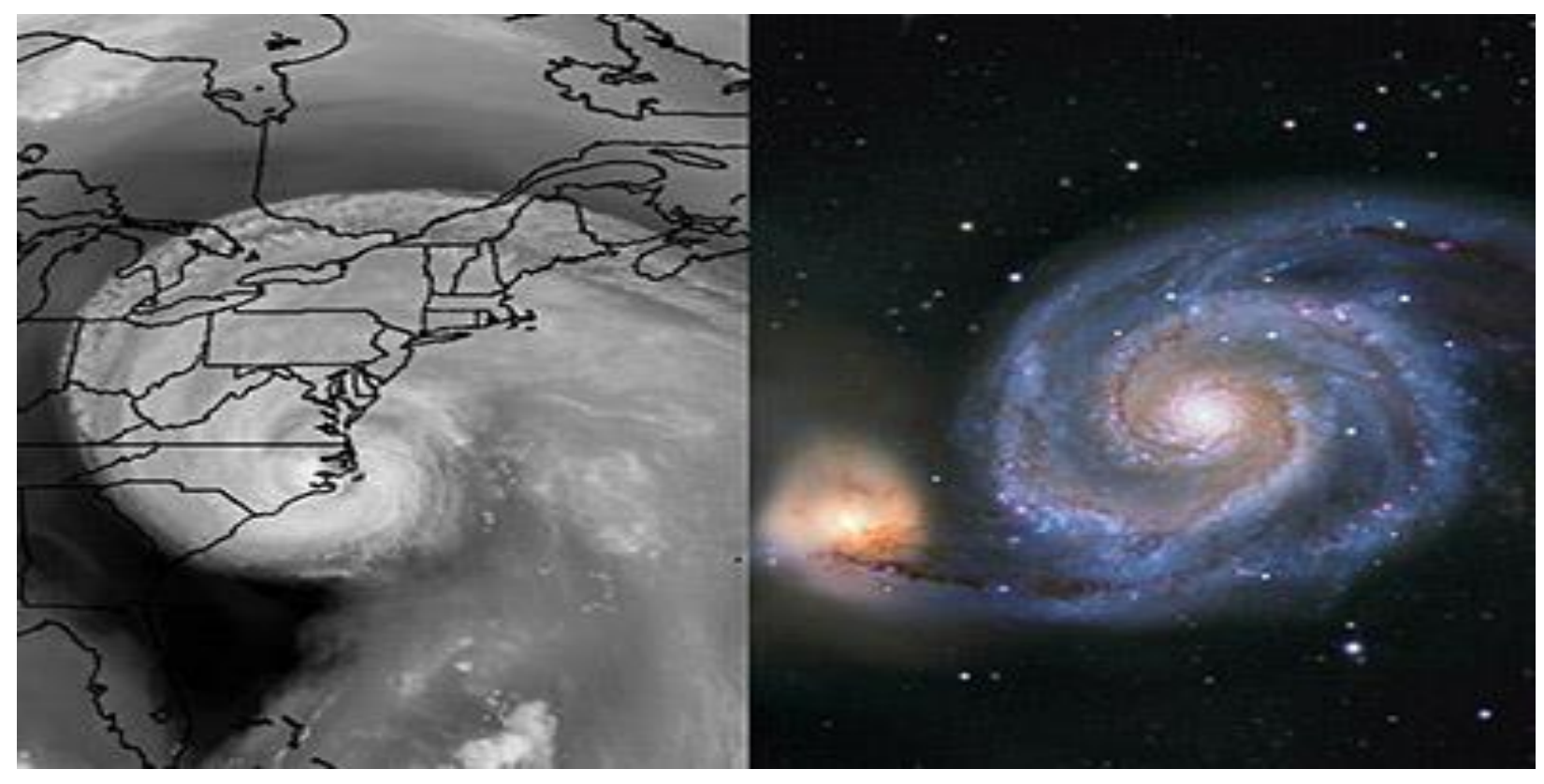

Figure 10: Comparison of Hurricane Isabel with spiral Whirpool Galaxy M51 (Image Credit to Lula 2003).

Galaxies' birth has several complex conditions which may bring a research topic (Loeb 2010). However, to the best of our knowledge, most of the spiral galaxies' theoretical models by 
purely analytic methods have no accurate results. As a result, the structure and evolution of the Universe (SEU) could not understand (Figure 11).

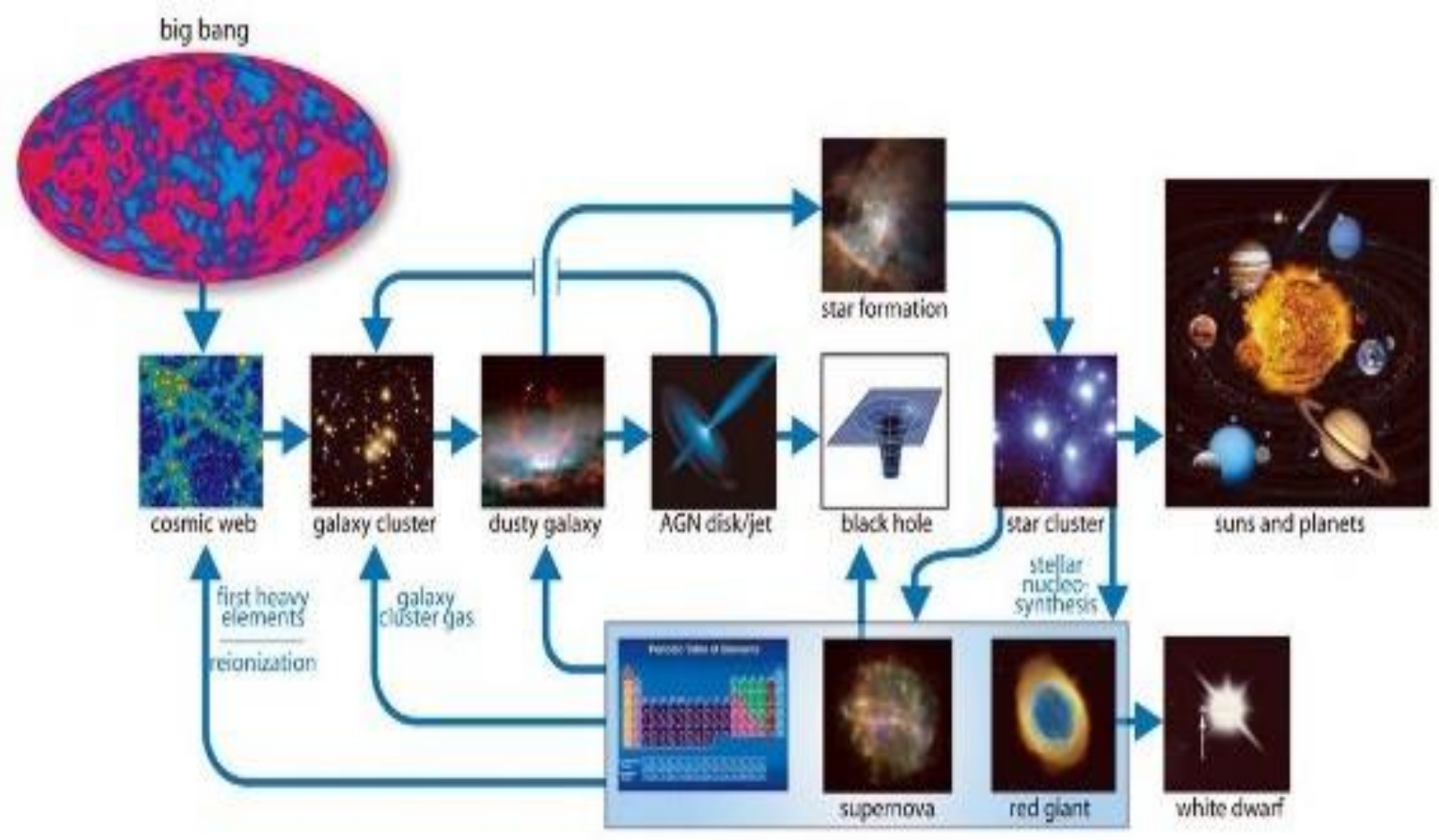

Figure 11: From the big bang to galaxies (Image Credit to NASA, CAS-Group 2021)

Hofmeister et al. compared the hurricanes with spiral galaxies (Figure 12) (Hofmeister, et al., 2017a; 2017b). In addition, Sofue et al. studied spiral galaxies' interior parts with early rotation curves measurements (Sofue et al., 2001). Moulton et al.'s observations are appropriate with the view long ago that galactic movements are controlled and embody the entire object's spin (Moulton 1970). 

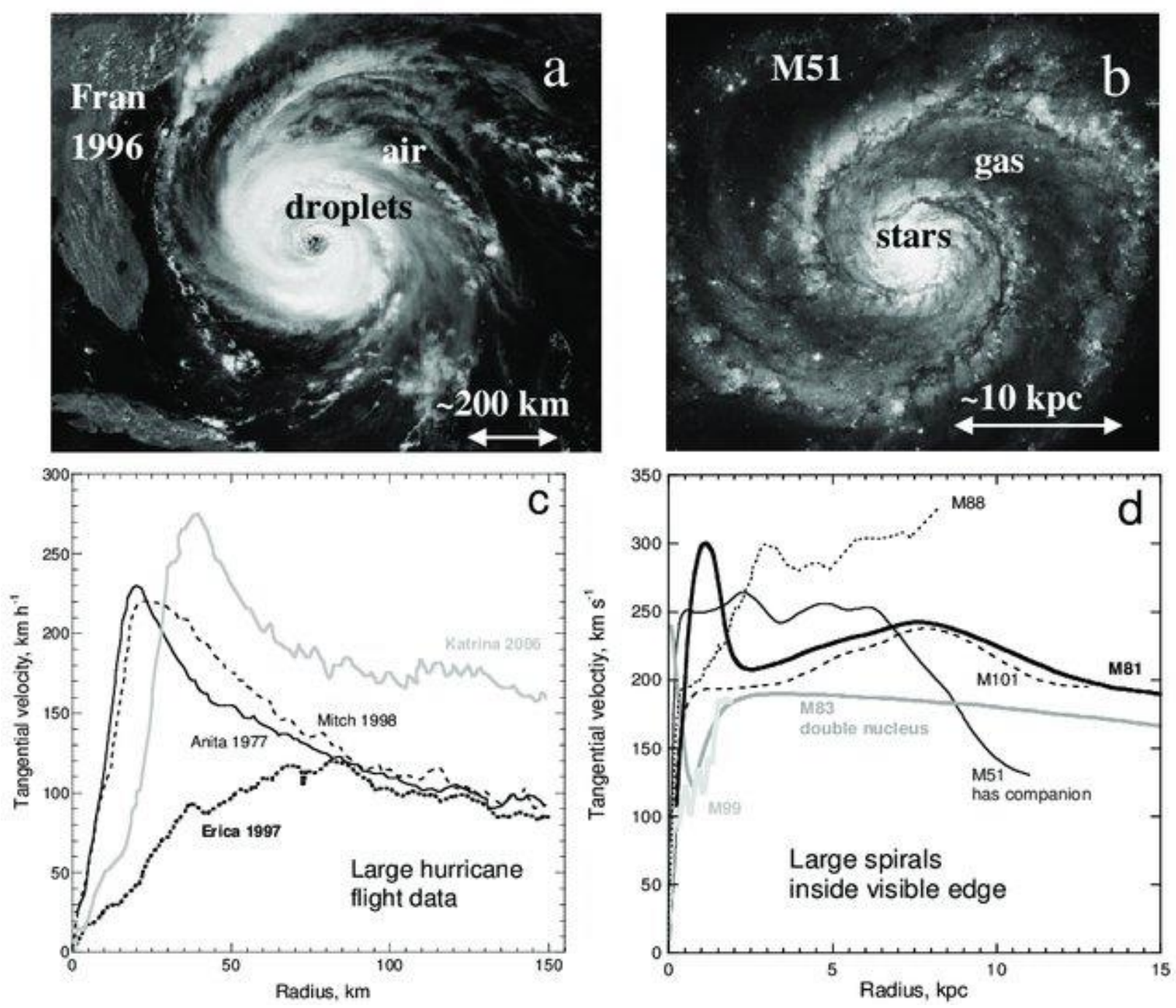

Figure 12: Comparison of hurricanes to spiral galaxies (Hofmeister and Criss 2017a).

A galaxy does rotate, but it is not this rotation that causes the spiral arms to form. Instead, the current theory for the formation of spiral arms is due to pressure waves. When these stars get old and eventually die, some will go supernova. This supernova will cause pressure waves in the interstellar medium to make the arms contract gasses. This contact will set off another wave of star formation, and the spiral shape will be perpetuated (Hofmeister, et al., 2020). Thus, gravity and 
angular momentum play an important role in the birth of hurricanes and galaxies. At the same time, they were confirming the similarities to the Golden Ratio (Frater et al., 2016).

\section{Conclusion}

There are several studies related to the comparative of Galaxies and Hurricanes by many curious researchers. Each of them focused on their perspectives. Nevertheless, unfortunately, both do not observe and compare and collect data easily. Especially, galaxies are the one has very fewer data compared to hurricanes. However, in this short review, we gave a couple of works to show the differences between hurricanes and galaxies.

A hurricane is much harder to solve a fluid mechanics problem than the simple Newtonian forces (at the macro-scale) that drive a galaxy. Hurricanes' centers are analogous to black holes at the spiral galaxies' centers, where the fastest rotation occurs. While we determine the galaxies by gravity, hurricanes can be determined by Earth's rotation and Sun's luminosity. Hurricanes formed with low-pressure wind systems and rotated in the center. Because of the low pressure, air rushing in to fill, which low pressure pulls inwards, highs push outwards. This effect is very different from what is going on in spiral galaxies. Gas pressure is not crucial on Galactic scales, not for stars that make up the spiral patterns.

As a result, hurricanes and galaxies are similar in shape and rotation. However, how they formed and what materials they have are the differences in each other. This comparison could be made with the advanced technology by using observational data in artificial intelligence in the near future. At that time, we can be sure that these two giant structures compare profound. 


\section{References}

Abdallah, H., Adam, R., Aharonian, F., Benkhali, F. A., Angüner, E. O., Arcaro, C., ... \& Noel, A. P. (2021). Search for dark matter annihilation in the Wolf-Lundmark-Melotte dwarf irregular galaxy with HESS. Physical Review D, 103(10), 102002.

Brunkard, J., Namulanda, G., \& Ratard, R. (2008). Hurricane katrina deaths, louisiana, 2005. Disaster medicine and public health preparedness, 2(4), 215-223.

Cao, Z., Liu, L., \& Zheng, T. (2018). Is the Spiral Galaxy a Cosmic Hurricane?. arXiv preprint arXiv:1802.02734.

Cas group and NASA (2021). Hurricanes and Galaxies - Surprising similarities in structure. Accessed October 21 2021. https://www.cas-group.net/similarities.htm

Chang, C. W., \& Feng, Y. (2021). Hurricane Bond Price Dependency on Underlying Hurricane Parameters. Asia-Pacific Journal of Risk and Insurance, 15(1), 1-21.

Collins, A. (2006). Violent weather: thunderstorms, tornadoes, and hurricanes. National Geographic Books.

Criss, R. E., \& Hofmeister, A. M. (2018). Galactic density and evolution based on the virial theorem, energy minimization, and conservation of angular momentum. Galaxies, 6(4), 115.

Criss, R. E., \& Hofmeister, A. M. (2020). Density profiles of 51 galaxies from parameter-free inverse models of their measured rotation curves. Galaxies, 8(1), 19. https://doi.org/10.3390/galaxies6040115. 
Davidge, T. J. (2021). New Blue and Red Variable Stars in NGC 247. The Astronomical Journal, $162(4), 152$.

Devaraj, J., Ganesan, S., Elavarasan, R. M., \& Subramaniam, U. (2021). A Novel Deep Learning Based Model for Tropical Intensity Estimation and Post-Disaster Management of Hurricanes. Applied Sciences, 11(9), 4129.

de Miguel, A. S., Zamorano, J., Aubé, M., Bennie, J., Gallego, J., Ocaña, F., ... \& Gaston, K. J. (2021). Colour remote sensing of the impact of artificial light at night (II): Calibration of DSLRbased images from the International Space Station. Remote Sensing of Environment, 264, 112611.

Dorminey, B. (2002). Cauldrons of Creation. In Distant Wanderers (pp. 1-18). Springer, New York, NY.

Dima, I., \& Desflots, M. (2010). Wind profiles in parametric hurricane models. Retrieved October, 19, 2013.

Emanuel, K. A. (1987). The dependence of hurricane intensity on climate. Nature, 326(6112), 483485.

English, N. (2014). The Spring Galaxies. In Grab'n'Go Astronomy (pp. 137-146). Springer, New York, NY.

Feng, J. Q. (2020). Rotating Disk Galaxies without Dark Matter Based on Scientific Reasoning. Galaxies, 8(1), 9. https://doi.org/10.3390/galaxies8010009.

Fernandez, J., Alonso, S., Mesa, V., Duplancic, F., \& Coldwell, G. (2021). Properties of galaxies 
with ring structures. Astronomy \& Astrophysics, 653, A71.

Frater, P., \& Duthie, A. B. (2016). Power scaling, vascular branching patterns, and the golden ratio. Ideas in Ecology and Evolution, 9(1), 15-18. https://doi.org/10.4033/iee.2016.9.4.n.

Galindo, I., Johnson, M. E., Martín-González, E., Romero, C., Vegas, J., Melo, C. S., ... \& Sánchez, N. (2021). Late Pleistocene Boulder Slumps Eroded from a Basalt Shoreline at El Confital Beach on Gran Canaria (Canary Islands, Spain). Journal of Marine Science and Engineering, 9(2), 138.

Gibbons, G. (2020). Hurricanes!(New Edition). Holiday House.

Gutro, Rob (2006). NASA Looks at Hurricane Cloud Tops for Windy Clues. Accessed October 21,2021. NASA. https://www.nasa.gov/vision/earth/lookingatearth/eyewall.html

Han, G., Ma, Z., Chen, D., Deyoung, B., \& Chen, N. (2012). Observing storm surges from space: Hurricane Igor off Newfoundland. Scientific reports, 2(1), 1-9.

Hofmeister, A. M., \& Criss, R. E. (2017a). Implications of geometry and the theorem of Gauss on Newtonian gravitational systems and a caveat regarding Poisson's equation. Galaxies, 5(4), 89.

Hofmeister, A. M., \& Criss, R. E. (2017b). The physics of galactic spin. Canadian Journal of Physics, 95(2), 156-166. https://doi.org/10.1139/cjp-2016-0625

Hofmeister, A. M., \& Criss, R. E. (2020). Debated Models for Galactic Rotation Curves: A Review and Mathematical Assessment. Galaxies, $8(2), \quad 47 . \quad 8 \quad$ (2) $\quad$ : 47. https://doi.org/10.3390/galaxies8020047

Houze, R. A., Chen, S. S., Smull, B. F., Lee, W. C., \& Bell, M. M. (2007). Hurricane intensity and 
eyewall replacement. science, 315(5816), 1235-1239.

Johnson, M. E., Guardado-France, R., Johnson, E. M., \& Ledesma-Vázquez, J. (2019). Geomorphology of a Holocene Hurricane deposit eroded from rhyolite sea cliffs on Ensenada Almeja (Baja California Sur, Mexico). Journal of Marine Science and Engineering, 7(6), 193.

Kaiser, R. I., Lauterwein, J., Müller, P., \& Roessler, K. (1992). Energy density effects in the formation of organic residues in frozen methane by $\mathrm{MeV}$ ions. Nuclear Instruments and Methods in Physics Research Section B: Beam Interactions with Materials and Atoms, 65(1-4), 463-467.

Kelvinsong, Ma (2012), The anatomy of a hurricane. Accessed October 212021. https://commons.wikimedia.org/wiki/File:Hurricane-en.svg

Kossin, J. P. (2017). Hurricane intensification along United States coast suppressed during active hurricane periods. Nature, 541(7637), 390-393.

Latif, M., Keenlyside, N., \& Bader, J. (2007). Tropical sea surface temperature, vertical wind shear, and hurricane development. Geophysical Research Letters, 34(1).

Lehnert, M. D., \& Heckman, T. M. (1996). Ionized gas in the halos of edge-on starburst galaxies: Evidence for supernova-driven superwinds. The Astrophysical Journal, 462, 651.

Li, E. (2016). Modelling mass distribution of the Milky Way galaxy using Gaia billion-star map. arXiv preprint arXiv:1612.07781.

Lineweaver, C. H., \& Egan, C. A. (2008). Life, gravity and the second law of thermodynamics. Physics of Life Reviews, 5(4), 225-242. 
Loeb, A. (2010). How did the first stars and galaxies form?. Princeton University Press.

Lula, Brian (2003). Logarithmic Spirals Isabel and M51. NASA, GHHC. Accessed October 21 2021. https://apod.nasa.gov/apod/ap030925.html

McDaniel, A., Jeltema, T., \& Profumo, S. (2021). X-ray shapes of elliptical galaxies and implications for self-interacting dark matter. Journal of Cosmology and Astroparticle Physics, 2021(05), 020.

Morishita, T. (2021). SuperBoRG: Search for the Brightest of Reionizing Galaxies and Quasars in HST Parallel Imaging Data. The Astrophysical Journal Supplement Series, 253(1), 4.

Moulton, F. R. (1970). An introduction to celestial mechanics. Courier Corporation.

NASA (2005), Hurricane Katrina, NASA. Accessed October 212021. https://earthobservatory.nasa.gov/images/15395/hurricane-katrina

NASA (2009). The Hubble Space Telescope. NASA Goddard Space Flight Center. Accessed October 20, 2021. https://asd.gsfc.nasa.gov/archive/hubble/

NASA (2014). NGC 4651: The Umbrella Galaxy. Photo Credit to R. Jay Gabany, Accessed October 21,2021. https://apod.nasa.gov/apod/ap140702.html

NASA (2019). Hubble Views a Galaxy with an Active Center. Accessed October 21,2021. https://www.nasa.gov/image-feature/goddard/2019/hubble-views-a-galaxy-with-an-active-center

NASA (2020). Hubble Sees Sculpted Galaxy. Accessed October 21,2021. https://www.nasa.gov/image-feature/goddard/2020/hubble-sees-sculpted-galaxy 
NASA (2020). Hubble Spies Sparkling Galaxy. Photo Credit to T. Armandroff, Accessed October 21,2021. https://www.nasa.gov/image-feature/goddard/2020/hubble-spies-sparkling-galaxy

NASA (2021). NASA/IPAC Extragalactic Database. Accessed July 1, 2021. http://ned.ipac.caltech.edu/.

NWS, National Wheather Service (2021). Hurricane Fran: September 5,1996. Accessed October 20, 2021. https://www.weather.gov/mhx/Sep051996EventReview

Ramage, C. S. (1959). Hurricane development. Journal of Atmospheric Sciences, 16(3), 227-237.

Sánchez, S. F. (2020). Spatially Resolved Spectroscopic Properties of Low-Redshift Star-Forming Galaxies. Annual Review of Astronomy and Astrophysics, 58, 99-155. https://doi.org/10.1146/annurev-astro-012120-013326.

Shah, H., \& Seta, A. (2021). Magnetic fields in elliptical galaxies: using the Laing-Garrington effect in radio galaxies and polarized emission from background radio sources. Monthly Notices of the Royal Astronomical Society, 508(1), 1371-1388.

Smith, H. E. (1999). The Milky Way Galaxy. University of California, San Diego. Accessed August 3, 2021. https://casswww.ucsd.edu/archive/public/tutorial/MW.html

Sofue, Y., Tutui, Y., Honma, M., Tomita, A., Takamiya, T., Koda, J., \& Takeda, Y. (1999). Central rotation curves of spiral galaxies. The Astrophysical Journal, 523(1), 136.

Sofue, Y., \& Rubin, V. (2001). Rotation curves of spiral galaxies. Annual Review of Astronomy and Astrophysics, 39(1), 137-174. 
Sofue, Y., Koda, J., Nakanishi, H., \& Onodera, S. (2003). The virgo high-resolution CO survey: II. Rotation curves and dynamical mass distributions. Publications of the Astronomical Society of Japan, 55(1), 59-74.

Suntola, T. (2013). Gestalting Structures in Physics. GESTALT THEORY, 35(1).

Stow, J. P., Bourassa, M. A., \& Holbach, H. M. (2020). Analyzing Gaps in Hurricane Rain Coverage to Inform Future Satellite Proposals. Remote Sensing, 12(17), 2673.

West, B. (2000). Mythologising a natural disaster in post-industrial Australia: The incorporation of cyclone Tracy within Australian national identity. Journal of Australian Studies, 24(66), 197204.

Williams, R. E. (1996). The Hubble Deep Field: Observations, data reduction, and galaxy photometry. arXiv preprint astro-ph/9607174.

Willoughby, H. E., \& Black, P. G. (1996). Hurricane Andrew in Florida: Dynamics of a disaster. Bulletin of the American Meteorological Society, 77(3), 543-550.

Willoughby, H. E., Darling, R. W. R., \& Rahn, M. E. (2006). Parametric representation of the primary hurricane vortex. Part II: A new family of sectionally continuous profiles. Monthly weather review, 134(4), 1102-1120. https://doi.org/10.1175/MWR3106.1.

Wu, C. C., Chou, K. H., Cheng, H. J., \& Wang, Y. (2003). Eyewall contraction, breakdown and reformation in a landfalling typhoon. Geophysical research letters, 30(17). 